\title{
Conservation of a Genetically Selected Broiler Line (42L)Using Cryopreserved Circulating Primordial Germ Cells (PGCs) Isolated by Filtration Method.
}

\author{
Atsushi Tajima ${ }^{* 1,2)}$, Guy F. Barbato ${ }^{3)}$, Takashi Kuwana ${ }^{4)}$ \\ and Roy H. Hammerstedt ${ }^{1)}$ \\ 1) Department of Biochemistry and Molecular Biology, Pennsylvania State University. \\ University Park, PA 16802, USA. \\ ${ }^{2)}$ Institute of Agriculture and Forestry, University of Tsukuba, \\ Tsukuba,Tsukuba, Ibaraki 305-8572, Japan. \\ ${ }^{3)}$ Department of Poultry Science. Pennsylvania State University. \\ University Park, PA 16802, USA. \\ 4) National Institute For Minamata Disease. Minamata, \\ Kumamoto 867-0008, Japan.
}

\begin{abstract}
A genetically selected broiler line (42L) developed at the Pennsylvania State University, PA, USA was used in the present study. In Experiment 1, embryonic blood was collected from chick embryos at stage 14-15 (Hamburger and Hamilton, 1951). Collected blood was suspended in Minimum Essential Medium (MEM) supplemented with $10 \%$ fetal bovine serum (FBS) and suspended blood sample was filtered using a nylon filter with 10 micrometer mesh opening. The number of PGC was measured before and after filtration and recovery rate was $39.4 \pm 6.5 \%$ (mean \pm sem). In Experiment 2, the viability of the filter-isolated frozen/thawed PGCs from a genetically selected broiler line (42L) was tested by transferring them into blood stream of the 2-day-old Brown Leghorn embryo.When semen from the male PGC recipient was tested on six different hens, $33 \%$ of a total of 216 chicks were derived from transferred PGCs. When the two female recipients received semen, $52 \%$ of the total of 21 chicks was derived from transferred PGCs. The results obtained in the present experiment indicate that it is possible to conserve poultry genetic resources using frozen/thawed circulating PGCs collected by a filtration method.
\end{abstract}

Key words : Filtration method, PGCs, cryopreservation, germ-line chimeras, chicken.

\section{Introduction}

It has been reported that the avian primordial germ cells (PGCs) originate from the central zone of the area pellucida (Ginsburg and Eyal-Giladi, 1987 ; Ginsburg and Eyal-Giladi, 1989 ; Kagami et al. 1997). PGCs then translocate to hypoblast and

Received : August 23, 2002 Accepted : October 22, 2002

Correspondence to Atsushi Tajima

Institute of Agriculture and Forestry, University of Tsukuba, Ibaraki 305-8572, Japan

Tel : 029-853-6688 Fax : 029-853-6205 E-mail : tajima@sakura.cc.tsukuba.ac.jp 
appear in the germinal crescent region at primitive streak stage ( Sutasurya et al. 1983 ; Ginsburg and Eyal-Giladi 1986). Thereafter, PGCs circulate temporarily within the whole embryo through the blood stream. The concentration of circulating PGCs are reported to be highest at around stage 14 (Tajima et al. 1999) and finally colonize in to gonadal anlage (Swift 1914 ; Fujimoto et al. 1976 ; Kuwana and Fujimoto, 1983 ; Ginsburg, 1994 ).

This unique mode of PGCs migration allowed to recover PGCs from germinal crescent (Shuman, 1981), blood (Yasuda et al. 1992) and gonadal ridge (Chang et al. 1995 ; Tajima et al. 1998).

The circulating PGCs have been isolated by using Ficoll density gradient centrifuge in domestic chickens and germ-line chimeras were produced by transferring unfrozen or frozen circulating PGCs (Tajima et al. 1993 ; Naito et al. 1994a). The recovery rate of circulating PGCs using Ficoll density gradient centrifuge is reported to be $3.8 \%$ (Yasuda et al. 1992). Apparently, the recovery rate of circulating PGCs needs improvement for efficient use of the eggs produced by the genetically important lines or breeds of chicken. The development of an alternative collection method of PGCs which is more efficient and practical was hence thought to be beneficial for the efficient production of germline chimeras.

In the present study, therefore, an alternative method of collecting circulating PGCs using nylon filter was used to isolate circulating PGCs from blood cells, and it was incorporated with cryopreservation protocol. Furthermore, the viability of the filter-isolated frozen/thawed PGCs from a genetically selected broiler line (42L) was tested by transferring them into blood stream of the 2-day-old Brown Leghorn embryo.

\section{Materials and Methods}

\section{Experiment 1.}

A genetically selected broiler line (42L), which was developed and maintained at The Pennsylvania State University, PA, USA, was used in the present study. The 42L broiler line was established by selecting low body weight individual at 42 days after hatch (Barbato, 1992).

Fertilized 42L eggs were incubated until they reached stage 14-15 (Hamburger and Hamilton, 1951) in an air-forced incubator ( $\mathrm{P}-008$, Showa Incubator Laboratory, Japan) maintained at $37.8^{\circ} \mathrm{C}$. Each egg was broken into a $9 \mathrm{~cm}$ plastic-dish (Fisher Scientific, Pittsburgh, PA) and embryonic blood was collected from dorsal aorta of the embryos using a fine glass pipette.

A fine glass pipette was prepared by pulling a $50 \mu \mathrm{l}$ capacity glass pipette (Cat \#2-000-050, Drummond Sci. CN, USA) with a micro-pipette puller (Narishige PA81-8811, Japan).

Blood collected from 5 embryos were pooled and placed in a $1.7 \mathrm{ml}$ capacity centrifuge tube (Costar \#3620, Corning, Corning NY) containing $100 \mu 1$ of Phosphate Buffered Saline without containing magnesium and calcium (PBS(-)).

Centrifuge tubes, pipette tips, and filters used in the present study were all coated with silicone using Sigma coat (SL-2, Sigma Chemicals, MO, USA) and autoclaved 
prior to use. Blood sample was thoroughly mixed using a fine glass pipette and PGCs were isolated using a $10 \mu \mathrm{m}$ mesh nylon filter $\left(\mathrm{E}^{-} \mathrm{CMN}-10\right.$, Small Parts, FL, USA). Number of PGCs before and after filtration was counted according to the method described previously (Tajima et al. 1999).

\section{Experiment 2.}

Blood collected from 5-10 embryos were pooled into $1.7 \mathrm{ml}$ capacity centrifuge tube containing $100 \mu l$ of Minimum Essential Medium (MEM, M-3516, Sigma Chemicals, St. Louis, MO) supplemented with 10\% FBS (A-1115-L, Hyclone Laboratory, Logan, UT) at room temperature. One hundred microliter of the diluted blood sample was transferred into sterile cryogenic vials (25723-1, Corning, Corning, NY) and cooled to $5^{\circ} \mathrm{C}$. This was followed by adding $100 \mu \mathrm{l}$ of MEM supplemented 20\% DMSO (D128-500, Fisher Scientific, Pittsburgh, PA) which were pre-cooled to $5^{\circ} \mathrm{C}$. The final concentration of DMSO in the sample, therefore, would be $10 \%(\mathrm{~V} / \mathrm{V})$.

The cryogenic vial was then placed into a freezing vessel (Bicell, Nihon Freezer Co. Japan), and it was placed into deep freezer maintained at $-80^{\circ} \mathrm{C}$ for $4 \mathrm{hrs}$. The temperature inside the freezing vessel is designed to decrease at $-1^{\circ} \mathrm{C} / \mathrm{min}$ when placed in $-80^{\circ} \mathrm{C}$. The cryogenic vial was then placed into liquid nitrogen $\left(-196^{\circ} \mathrm{C}\right)$ and stored for 2 to 4 weeks.

The sample was thawed in ice water followed by adding $100 \mu l$ of MEM containing 5\% DMSO (MEM-5D).

\section{Filtration :}

PGCs were isolated using a $10 \mu \mathrm{m}$ mesh nylon filter. First, $50 \mu \mathrm{l}$ of MEM-5D was placed to humidify the filter followed by placing $50 \mu l$ each of the frozen/thawed blood sample until all the sample were filtered. After all the sample were filtered, nylon filter was flushed by placing $50 \mu l$ of MEM-5D, followed by $50 \mu l$ of MEM supplemented with 2.5\% DMSO (MEM-2.5D) and finally $50 \mu l$ of MEM without containing DMSO. Upon completion of the filtration process, the filter was removed using a pair of forceps and placed into a $1.7 \mathrm{~m} l$ capacity centrifuge tube containing $50 \mu l$ of MEM. The centrifuge tube containing a filter was mixed using a vortex mixer. After mixing, the filter mesh was placed to the area adjacent to the cap of the centrifuge tube, so that the mesh would not touch the fluid surface during centrifuge, and centrifuged at $700 \mathrm{~g}$ for 10 minutes.

\section{PGC Transfer}

After centrifuge, cell suspension was placed in the $35 \mathrm{~mm}$ petri-dish, covered with mineral oil (JT 2705-1, JT Baker Inc, Phillipsburg, NJ) to avoid the evaporation of the medium. Approximately 30-70 PGCs, as judged by the morphology, were picked up into fine glass pipette and injected into the dorsal aorta of the windowed egg containing Brown Leghorn (BL) embryos at stage 14 or 15 of development. The diameter of the window on the egg was approximately $1.5 \mathrm{~cm}$. The fertilized BL eggs were kindly provided by the courtesy of DeKalb, Ill, USA. Windowed eggs containing BL embryos were prepared 3 to 4 hours prior to germ cell injection. During this period, approximately 4 to $6 \mu l$ of the embryonic blood was removed according to the method described previously (Naito et al. 1994b). The blood removal was carefully made so that no 
blood was observed to leak from embryos after removing the glass pipette. The purpose of removing blood from the recipient embryos was to eliminate endogenous circulating PGC (Naito et al. 1994b).

After germ cell injection, the window was sealed with plastic tape (Cat \#23-8043, ScotchTM Super Strength Mailing Tape, 3M, USA) and the embryo was allowed to develop until hatching. One male chick and two female chicks were produced. Hatched chicks were raised beyond sexual maturity so that progeny-testing could be performed. For female PGC recipients, $50 \mu l$ of pooled semen collected from 42L rooster were inseminated weekly. On the other hand, semen was collected from a male PGC recipient once a week and $50 \mu l$ of undiluted semen was inseminated to four $42 \mathrm{~L}$ females per male from $1^{\text {st }}$ through $5^{\text {th }}$ hatch. A male PGC recipient was subjected to molt after completing $5^{\text {th }}$ insemination and eight new $42 \mathrm{~L}$ females were used to inseminate from $6^{\text {th }}$ through $8^{\text {th }}$ hatch.

The BL carries the homozygous dominant brown pigment gene $(\mathrm{C} / \mathrm{C})$ whereas 42 $\mathrm{L}$ broiler line used in the present study carries the homozygous recessive brown pigment gene $(\mathrm{c} / \mathrm{c})$. Therefore, the identity of the spermatozoa or ova produced from germ-line chimeras can be ascertained by observing the feather color of the newly hatched chicks.

\section{Results}

\section{Experiment 1}

Recovery rate of the morphologically normal circulating PGCs was $39.4 \pm 6.5 \%$ (means \pm sem)(Table 1). The recovery rate of the circulating PGCs using a newly developed filtration method was considered to be adequate for the practical purpose, even though the recovery rate of PGCs fluctuated among replicates.

\section{Experiment 2.}

Primordial germ cells were injected into 28 recipient eggs, and 3 chicks (1male and 2 females) were hatched.

Result of the progeny testing for the male PGC recipient was shown in Table 2. In

Table 1. Recovery of circulating PGCs from 42L embryo at stage 14-15 using filtration

\begin{tabular}{|c|c|c|c|c|}
\hline Sample \# & Stage & $\begin{array}{l}\text { Number of } \\
\text { PGCs applied }\end{array}$ & $\begin{array}{c}\text { Number of } \\
\text { PGCs recovered }\end{array}$ & $\begin{array}{c}\text { Recovery rate } \\
(\%)\end{array}$ \\
\hline 1 & 14 & 83 & 38 & 45.6 \\
\hline 2 & 15 & 100 & 28 & 28.0 \\
\hline 3 & 15 & 17 & 7 & 42.0 \\
\hline 4 & 14 & 100 & 59 & 59.0 \\
\hline 5 & 15 & 67 & 15 & 22.5 \\
\hline Average \pm SEM & - & $73.3 \pm 15.5$ & $29.4 \pm 9.1$ & $39.4 \pm 6.5$ \\
\hline
\end{tabular}


a male PGC recipient, fertility trial was conducted for 8 weeks and the overall ratio of white chicks were 33\% (72/216).

Weekly ratio of the white chicks for a male PGC recipient during progeny testing were 50.0\% (6/12), 47.6\% (10/21), 30.8\% (8/26), 41.7\% (5/12), 30.0\% (6/20), $30.0 \%(9 / 30), 32.7 \%(17 / 52)$ and $25.6 \%(11 / 43)$, for hatch number $1,2,3,4,5,6,7$ and 8 , respectively.

Result of the progeny testing for 2 female PGC recipients were shown in Table 3.

In female 1, the progeny testing was for 7 hatches and the average ratio of white chicks was $52.6 \%(10 / 19)$. The weekly ratios of the white chicks against brown chicks were $100 \%(4 / 4), 100 \%(4 / 4), 50 \%(1 / 2), 25 \%(1 / 4), 0 \%(0 / 0), 0 \%(0 / 0)$ and $0 \%$ $(0 / 5)$ for hatch number $1,2,3,4,5,6$ and 7 , respectively.

In female 2, progeny testing was conducted for 5 weeks and the average ratio of white chicks was $50.0 \%(1 / 2)$. The weekly ratios of the white chicks against brown chicks were $0 \%(0 / 0), 100 \%(1 / 1), 0 \%(0 / 0), 0 \%(0 / 1)$ and $0 \%(0 / 0)$ for week $1,2,3,4$ and 5 , respectively.

All hatched white chicks were weighed at hatch, 14 and 42 days during the grow out phase and moved to a breeder house and maintained as described (Barbato, 1992).

The growth curve of the all white chicks during the test period were identical to the growth curve of the typical 42L broiler line as described in Barbato (1992) (data not shown). Furthermore, all birds displayed white mature feather color at 16 weeks of age without any black flecks or brown backs.

\section{Discussion}

It is apparently true that the isolation of PGCs is the first and the most important step for the production of germline chimera in avian species. The Ficoll density gradient centrifuge has been primarily used to isolate circulating PGCs in avian species

Table 2. Result of the progeny testing for a male PGC recipient

\begin{tabular}{ccccc}
\hline \hline Hatch number & $\begin{array}{c}\text { Age of male } \\
\text { (Week) }\end{array}$ & $\begin{array}{c}\text { \#Total chick } \\
\text { Hatched }\end{array}$ & $\begin{array}{c}\text { \#of white } \\
\text { chicks }\end{array}$ & $\begin{array}{c}\text { White chicks } \\
(\%)\end{array}$ \\
\hline 1 & 30 & 12 & 6 & 50.0 \\
2 & 31 & 21 & 10 & 47.6 \\
3 & 32 & 26 & 8 & 30.8 \\
4 & 33 & 12 & 5 & 41.7 \\
5 & 34 & 20 & 6 & 30.0 \\
6 & 40 & 30 & 9 & 30.0 \\
7 & 41 & 52 & 17 & 32.7 \\
8 & 42 & 43 & 11 & 25.6 \\
\hline Totals & & 216 & 72 & 33.3 \\
\hline
\end{tabular}

Note: A male was subjected to molt after completing $5^{\text {th }}$ insemination.

Four hens were used from $1^{\text {st }}-5^{\text {th }}$ hatch, and eight new hens were used from $6^{\text {th }}$ through $8^{\text {th }}$ hatch 
(Naito et al. 1994a ; Naito et al. 1994b ; Naito et al. 1998 ; Tajima et al. 1993 ; Yasuda et al. 1992). However, the recovery rate of circulating PGCs using Ficoll density gradient centrifuge remained to be $3.8 \%$ (Yasuda et al. 1992). Furthermore, it has been suggested that PGCs swell slightly after Ficoll density gradient (personal communication).

The advantage of using filtration method over Ficoll density gradient, on the other hand, was considered to be 1) higher recovery rate of PGCs 2) isolation process of PGCs can be simplified 3) Stress applied to PGCs during isolation process is minimal. Simplification of isolation process is especially true when cryopreserved PGCs are used, as in the case of the present study, since the DMSO removal and the PGC isolation process can be performed simultaneously.

Prior to filtrate the blood sample after thawing, equal volume of a MEM-5D was added to the sample so that the concentration of DMSO was reduce to $7.5 \%$. Furthermore, MEM-5D was initially used to flush the thawed blood followed by flushing the sample using MEM without containing DMSO.

From the preliminary experiment, stepwise decrease of the DMSO concentration in the flushing medium was found to be important to prevent PGCs from excessive

Table 3.

(A) Result of the progeny testing for female-1

\begin{tabular}{ccccc}
\hline \hline Hatch number & $\begin{array}{c}\text { Number of } \\
\text { eggs set }\end{array}$ & $\begin{array}{c}\text { \#Total chick } \\
\text { Hatched }\end{array}$ & $\begin{array}{c}\text { \#of white } \\
\text { chicks }\end{array}$ & $\begin{array}{c}\text { White chicks } \\
(\%)\end{array}$ \\
\hline 1 & 9 & 4 & 4 & 100.0 \\
2 & 13 & 4 & 4 & 100.0 \\
3 & 14 & 2 & 1 & 50.0 \\
4 & 11 & 4 & 1 & 25.0 \\
5 & 5 & 0 & - & - \\
6 & 14 & 0 & - & 0.0 \\
7 & 6 & 5 & 0 & 52.6 \\
\hline Totals & 72 & 19 & 10 & \\
\hline
\end{tabular}

(B) Result of the progeny testing for female-2

\begin{tabular}{ccccc}
\hline \hline Hatch number & $\begin{array}{c}\text { Number of } \\
\text { eggs set }\end{array}$ & $\begin{array}{c}\text { \#Total chick } \\
\text { Hatched }\end{array}$ & $\begin{array}{c}\text { \#of white } \\
\text { chicks }\end{array}$ & $\begin{array}{c}\text { White chicks } \\
(\%)\end{array}$ \\
\hline 1 & 2 & 0 & - & - \\
2 & 1 & 1 & 1 & 100.0 \\
3 & 0 & 0 & - & - \\
4 & 2 & 1 & 0 & 0.0 \\
5 & 1 & 0 & - & - \\
\hline Totals & 6 & 2 & 1 & 50.0 \\
\hline
\end{tabular}

Note : Female- 2 died after completing $5^{\text {th }}$ hatch. 
swelling and even explosion due to the hypo-osmotic effect (data not shown). The morphology of the isolated PGCs after filtration was evaluated as normal both before and after freezing. A $10 \mu \mathrm{m}$ filter was used in the present study to isolate PGCs from blood cells since the diameter of the circulating PGCs are approximately $14 \mu \mathrm{m}$ (Fujimoto et al. 1976) whereas the diameter of the blood cells are $7 \mu \mathrm{m}$ (unpublished observations). The recovery efficiency of the PGCs using a nylon mesh with smaller opening such as 7 or $8 \mu \mathrm{m}$ should be tested in the future.

Recovery rate of the PGCs fluctuated considerably among replicate. The reason for the fluctuation of the recovery rate remains unclear but one of the reasons could be due to the slight distortion created on the filter surface upon mounting the sample which resulted in change in the uneven mesh opening during filtration process. Alternatively, size of the mesh opening might have slightly changed during autoclaving even though the mesh is claimed to tolerate up to about $200^{\circ} \mathrm{C}$. Therefore, each process of the filtration process should be critically evaluated to stabilize and to improve the recovery rate of the PGCs. The recovery rate of PGCs could also be improved by adjusting the combination between mesh size of the filter and the osmotic pressure of the medium.

Nevertheless, it was shown in the present experiment that the morphologically normal PGCs could be isolated repeatedly using a filtration method. Therefore, a transplantation study was conducted to evaluate the quality of the isolated PGCs using the filtration method.

From the result obtained from the PGC transplantation study in Experiment 2, it was shown that the frozen/thawed PGCs isolated by a filtration method was confirmed to be physiologically and functionally normal PGCs. The proportion of the white chicks produced from a male germline chimera remained within a range between 26 to $50 \%$ during 8 hatches of progeny testing. On the other hand, the proportion of the white chicks produced from female germline lowered considerably as the progeny testing progressed. The reason for the considerable week to week variation in the proportion of the white chicks in a female PGC recipient remain unsolved but one of the reason could be related to the recruitment process of the primary follicle in the ovary.

Growth curve of the white chicks at hatch, 14 and 42 days as well as feather color at 16 weeks of age were all typical to $42 \mathrm{~L}$ broiler line. These results show that the white chicks hatched from germ line chimeras (Brown Leghorn) were all pure 42L broiler line.

Therefore, it was concluded in the present study that the filtration method is an effective and practical means to recover highly viable circulating PGCs in domestic chicken.

Future study should include to inseminate female germline chimera with frozen thawed semen, which should be a practical means to preserve genetically important genetic resources in avian species.

\section{Acknowlegment}

Brown Leghorn eggs used in the study were provided by Dr. Larry Vint of DeKalb Poultry Research. Appreciation is expressed for assistance provided by the Poultry 
Teaching and Research Facility of The Pennsylvania State University. Dr. Edward G. Buss and Dr. Rupert P. Amann for their critical advice in conducting and preparing the manuscript.

The present study was supported in part by a grant from the Ministry of Education, Science, Culture and Sport, Japan (09460123) to AT, and support from the Pennsylvania Agricultural Experiment Station to GFB.

\section{References}

Barbato GF. Divergent selection for exponential growth rate at fourteen or forty two days of age. 1. Early responses. Poultry Science, $71: 1985-1993.1992$.

Chang IK, Tajima A, Chikamune T, Ohno T. Proliferation of chick primordial germ cells cultured on stroma cells from the germinal ridge. Cell Biology International Report, 19 : 143-149. 1995.

Fujimoto T, Ukeshima A, Kiyofuji R. The origin, migration and morphology of the primordial germ cells in the chick embryo. Anatomical Record, 185 : 139-153. 1976.

Ginsburg M. Primordial germ cell formation in birds. Ciba Foundation Symposium, 182 : 52-61. 1994.

Ginsburg $\mathbf{M}$ and Eyal-Giladi $\mathrm{H}$. Temporal and spatial aspects of the gradual migration of primordial germ cells from the epiblast into the germinal crescent in the avian embryo. Journal of Embryology and Experimental Morphology, 95 : 53-71. 1986.

Ginsburg $\mathrm{M}$ and Eyal-Giladi H. Primordial germ cells of the young chick blastoderm originate from the central zone of the area pellucida irrespective of the embryo-forming process. Development, $101: 209-219.1987$.

Ginsburg M and Eyal-Giladi H. Primordial germ cell development in cultures of dispersed central disks of stage X chick blastoderms. Gamete Research, 23 : 421-427. 1989.

Hamburger V and Hamilton HL. A series of normal stages in the development of chick embryo. Journal of Morphology, $88:$ 49-92. 1951.

Kagami H, Tagami T, Matsubara Y, Harumi T, Hanada H, Maruyama K, Sakurai M, Kuwana T, Naito M. The developmental origin of primordial germ cells and the transmission of the donor-derived gametes in mixed-sex germline chimeras to the offspring in the chicken. Molecular Reproduction and Development, 48 : 501-510. 1997.

Kuwana $\mathrm{T}$ and Fujimoto $\mathrm{T}$. Active locomotion of human primordial germ cells in vitro. Anatomical Record, $205: 21-26.1983$.

Naito M, Tajima A, Tagami T, Yasuda Y, Kuwana T. Preservation of chick primordial germ cells in liquid nitrogen and subsequent production of viable offspring. Journal of Reproduction and Fertility, $102: 321-325$. 1994a.

Naito M, Tajima A, Yasuda Y, Kuwana,T. Production of germline chimeric chickens, with high transmission rate of donor-derived gametes, produced by transfer of primordial germ cells. Molecular Reproduction and Development, 39 : 153-161. 1994b.

Naito M, Tajima A, Yasuda Y, Kuwana T. Donor primordial germ cell-derived offspring from recipient germline chimaeric chickens : absence of long-term immune rejection and effects on sex ratios. British Poultry Science, 39 : 20-23. 1998.

Shuman RM. Primordial germ cell transfer in the chicken, Gallus domesticus. In : M.S. Thesis. St.Paul : University of Minnesota. 1981.

Sutasurya LA, Yasugi S, Mizuno T. Appearance of primordial germ cells in young chick blastoderms cultured in vitro. Development Growth and Differentiation, 25 : 517-521. 1983.

Swift $\mathrm{CH}$. Origin and early history of the primordial germ cells of the chick.American Journal of Anatomy, $15: 483-516.1914$.

Tajima A, Naito M, Yasuda Y, Kuwana T. Production of germ line chimera by transfer of primordial germ cells in domestic chicken (Gallus domesticus). Theriogenology, 40 : 509519. 1993.

Tajima A, Naito M, Yasuda Y, Kuwana T. Production of germ-line chimeras by transfer of 
cryopreserved gonadal primordial germ cells (gPGCs) in chicken. Journal of Experimental Zoology, $280: 265-267.1998$.

Tajima A, Hayashi H, Kamizumi A, Ogura J, Kuwana T, Chikamune T. Study on the concentration of circulating primordial germ cells (cPGCs) in early chick embryos. Journal of Experimental Zoology, $284:$ 759-764. 1999.

Yasuda Y, Tajima A, Fujimoto T, Kuwana T. A method to obtain avian germ-line chimaeras using isolated primordial germ cells. Journal of Reproduction and Fertility, $96: 521-52$. 1992. 